\title{
Overcoming interference in short-term memory through distinctive mental contexts'
}

\author{
GERALD S. BLUM, JED R. GRAEF, AND LOUISE S \\ HAUENSTEIN, UNIVERSITY OF MICHIGAN, Ann Arbor, \\ Michigan
}

An exploratory series of investigations was carried out with a very experienced hypnotic $S$ to determine whether manipulations of his mental context could lead to mastery of a seemingly impossible recall task, i.e., lists of six trigrams all drawn from the pool of letters JKQWXZ. Dramatic successes were eventually achieved when prior hypnotic programming served to fractionate the contexts within which trigrams were perceived and remembered. Transfer of this acquired capacity to insulate items contextually was noted in the waking state.

In an earlier study we were impressed by the finding that distinctive mental contexts exert a powerful effect upon subsequent salience of material registered in those contexts. Strings of consonants presented under hypnosis were more likely to "pop into mind" spontaneously later in the hypnotic state than in the waking; conversely stimuli registered in the waking state were more salient afterward in waking than hypnosis (Blum, 1967). Some explorations of the unusual persistence of content acquired in unique hypnotic settings over periods of months and even years further reinforced our desire to test the limits of context manipulation as an aid in surmounting obstacles to short-term recall.

Method. We chose as the $\mathrm{S}$ for this investigation a veteran of a great deal of research involving hypnosis, a male undergraduate who had also participated in the studies mentioned above. The task which we sought to train this admittedly atypical $S$ to master was a seemingly impossible one. A trial consisted of the exposure of six consonant trigrams, all taken from the same pool of letters-JKQWXZ. These particular trigrams were selected for their qualities of maximal interference and minimal association value. Each trigram was shown in a tachistoscope for $1 \mathrm{sec}$ followed by a 4-sec interval devoted to its rehearsal. A metronome was beating 1 per sec throughout the trial to assist in regularizing the rehearsal. Four sec after the sixth trigram's rehearsal, $S$ was asked to recall all the items to the best of his ability and to indicate when he was resorting to guesswork in order to produce six responses. He was free to take as much time as he wanted, and there were no requirements for recalling the trigrams in any particular sequence. Approximately $20 \mathrm{sec}$ elapsed between the end of S's recall and presentation of the first trigram on the subsequent trial. We began with 12 different lists which were presented in random order throughout blocks of six trials separated by rest periods. No more than four blocks were included in any given 2-h session. Two sample lists follow: WQX QJK ZWJ XKZ KQW ZXQ; KJQ JKZ WKX QZW ZJK WQJ.

For scoring purposes the decision was made to give half-credit ( 1 point) for a report containing all three letters of a stimulus trigram but not in their original order, or two of the three letters both in correct position. Correctly recalled items are given two points, so that a perfect score on a trial is 12 . Guesses are scored the same way and included in the total.

The task of achieving perfect scores seemed fittingly formidable in practice as well as theory when we tried it ourselves and found that we had to struggle to get even three items right. We instructed $S$ at the outset to make use of the techniques which he had acquired in his many hours of participation in salience experiments: attending to and rehearsing each trigram in turn without trying to keep previous items in mind during the stimulus presentation; and letting them just come to mind during recall. With this set he was able to average around 7 points, which usually indicates three trigrams correct and one partly correct out of the six. Recency was most in evidence, the sixtl item being remembered best and the fifth next best. Our efforts to improve on this baseline extended over almost two months, with $\mathbf{S}$ coming in for two sessions a week.

Results. The first context manipulation was intended to split the list into two parts. The $\mathrm{S}$ was programmed previously under hypnosis to enter a mentally alert state of hypnosis in response to a signal before the first trigram was shown. This hypnotic context automatically terminated, with posthypnotic amnesia, after rehearsal of the third trigram, and $S$ proceeded to view and rehearse the last three items while awake. After three trigrams were reported in the waking state, $S$ automatically returned to mentally alert hypnosis (amnesia no longer in effect) for the report of three more. Scores improved slightly to a mean of 8 but scores in the waking control condition increased virtually the same amount. Switching the two halves in various orders during registration and recall either did not help or actually hindered performance. Nor did other attempts succeed. For example, a posthypnotic instruction to "link" each item to its serial position was not a significant aid to recall. Hallucinating the third trigram in red and the fourth as extra large did not turn the trick. Programming a momentary "totally blank mind" after each rehearsal in order to separate more sharply consecutive items in the list produced a marked decrement in scores. After a number of such efforts had failed, we seriously considered abandoning the project.

However, a major breakthrough occurred when we decided to fractionate $S$ 's mental states. He had generally been more successful with items from the last half of the list, especially the sixth and fifth, so the strategy dictated a unique context for each of the first three trigrams. Additional insulation was to be provided by inducing amnesia for each mental context and its trigram immediately after rehearsal, with lifting of the amnesia to occur at the instant $S$ returned to that context during recall. Accordingly, he was programmed hypnotically to be "deep asleep" (hypnosis) while looking at and rehearsing the first trigram; "half awake, half asleep" for the second; and in a posthypnotic "dream" state (distinguished by its "floating quality" in S's words) for the third. Thereupon he returned to the waking state for the last half of the presentation and remained awake while recalling three items, usually the sixth. fifth, and fourth in that order. At that point he automatically (by prior instruction, of course) entered hypnosis and recalled the first trigram; emerged to a semihypnotic state, recalling the second: and switched to the postyhpnotic dream state in order to remember the third. Each of the three items was thus imbedded in a unique mental setting and the normal processes of interference proved to be overcome. With this procedure $S$ produced on several occasions an amazing string of successes. He was virtually perfect on six consecutive trials (score of 71 out of a possible 72) three times in two sessions. Two of these runs came in the first block of a day's trials when he was fresh but the other took place in the middle of a session. The overall mean score for 48 trials in these two sessions was 10.36 (more than five of the six trigrams typically recalled).

Most impressive to us as observers was the seeming resistance of the contextually stored items to the passage of time. For example, on a given trial $S$ might recall the sixth and fifth quickly and then deliberate as long as a minute or two over the fourth. After guessing at the fourth, often incorrectly, he would then breeze through the first, second, and third without hesitation. On many occasions the third trigram, theoretically one of the most difficult to remember and appearing last in his programmed recall order, popped into mind immediately even after three or four min had passed since its original presentation. The fact that he had stumbled over several of the other items seemed to have no bearing on the easy availability of the third as soon as he slipped into the unique "dream" context where that item was the only one registered.

Waking control trials had been suspended in these sessions while concentrating on the several differentiated mental contexts programmed previously under hypnosis. When success was achieved with this method we resumed the control trials and discovered, to our surprise, that he was now able to do just as well in the absence of hypnotic instruction-strings of perfect trials and an overall mean score above 10 . The only difference was that, in the absence 
of programming, the order of recall was typically 6-5-4-3-2-1 instead of 6-5-4-1-2-3. Our first thought was that somehow, over the course of endless trials with the 12 lists, he had learned the 72 trigrams despite their difficulty and overlap. Such an incredible feat was soon discounted when we constructed new equivalent lists of trigrams (again taken from the pool $\mathrm{JKQWXZ}$ ) and his performance did not suffer. The mystery of this dramatic improvement in waking control scores was cleared up at least partially in subsequent inquiries, which will be taken up in the discussion below.

Before concluding the empirical work, however, we felt obliged to meet the objection that $\mathrm{S}$ might have been using all kinds of associative mnemonic devices to learn the trigrams, despite our explicit instructions not to do so and his own statements to the contrary. Accordingly, we carried out a control series in which he was told to form as many associations as possible to the six trigrams in a list. He found the task extremely difficult (at most only a couple of associations suggested themselves on a trial, e.g., "crazy" for KZJ; "jacks" for JQX), and the scores dropped off.

Discussion. After the experiments were over, inquiries were conducted in both the waking and hypnotic states. While awake $S$ is amnesic for the hypnotic programming procedures, so his comments mainly described how he usually ignores previous items in the list during the presentation; the fact that he just "sits and waits" for the recall signal during the 4-sec pause after the sixth trigram's rehearsal (occasionally the sixth and/or fifth pop into mind then); and his attempts to recall systematically in reverse order ("sometimes I remember the first but $I$ save it to the end to keep from getting confused by the mixed order").

In the subsequent hypnotic inquiry, where amnesia was not present for either hypnotic or waking material, he reported that the hypnotically programmed trials differ in that the list "seems more chopped up into individual pieces." During both the presentation and recall phases he is completely unaware of trigrams from other mental contexts while immersed in a particular one. Replying to a question about possible changes in his response to the waking control trials in the course of the sessions, he stated that now he is able to proceed in a "more orderly" fashion in the recall by going backwards from sixth to first. Next he volunteered the interesting opinion that the programmed trials, in which the list is broken into small parts, probably helped the ensuing control trials ("it gives each trigram a particular place; when I see the first trigram I also register the fact that it's the first, and the same with the second and third").

Thus it seems that the hypnotically programmed mental contexts served to insulate the imbedded trigrams sufficiently from one another to render interference minimal during registration, storage, and recall. Reviving a mental context in the recall phase appeared to elevate the salience of its trigram to the exclusion of all others. It is unlikely that the accompanying amnesia was solely responsible because a rather similar separation of items during presentation by inserting the totally blank mind condition had failed. S's actual experience of the mental context seems to have been vital to the insulation process, for unique associative settings in themselves had no appreciable effect (hallucinating the third trigram in red or the fourth as extra large). However, a definitive statement comparing the efficacy of distinctive mental contexts to less extreme contextual variations must await more systematic study. We also know from S's statements, substantiated by the empirical check, that he was not forming outside associations to the consonants themselves as mnemonic aids.

From both data and hypnotic inquiry it is apparent that $\mathrm{S}$ was able to extend to the waking state his newly acquired skill in retaining and manipulating each trigram as a distinct entity. During recall he could mentally set aside with confidence the spontaneously intruding first trigram, for example, secure in the knowledge that he could bring it back at the appropriate point in the order. The process by which this waking extension took place is not obvious but we do have S's implication that the position of each trigram somehow substituted for the mental state in providing a unique context. Having once learned a technique for insulating items, it becomes relatively simple to attach a distinctive identification tag, e.g., position in sequence, to the separate entities. The prior necessity of learning such a technique, achieved through practice in experiencing the various mental states, is demonstrated by the fact that an earlier hypnotic instruction to link each trigram with its serial position had been unsuccessful. In order for the link to become effective finally, $S$ first had to master the insulation technique. Another factor contributing to the eventual waking success may have been his increased attention to the serial positions as such, growing out of the programmed necessity for changing mental states in advance of each of the first four trigrams. There is also a remote possibility that simply the additional amount of practice itself suddenly resulted in higher scores for both programmed and waking trials. Though this alternative cannot be entirely discounted because of the absence of relevant control data, it is very unlikely that practice for a month and a half could be ineffective and yet be followed by a marked spurt for no other reason.

By inference from these rather dramatic findings with an $S$ optimally trained for the purpose, it seems reasonable to suppose that naturally occurring, distinctive mental contexts, such as sleep, waking, and intervening stages of reverie, can exert similarly selective influences upon the memory process.

\section{REFERENCE}

BLUM, G. S. Experimental observations on the contextual nature of hypnosis. Intern. J. Clin. Exp. Hypn., 1967, 15, 160-171.

NOTE

1. This research was supported by NIMH Grant No. 08847-03. 